\title{
Mobilizing peptides in immunity
}

\author{
Harnessing the immune system to treat disease will be facilitated by a greater understanding of the \\ origins and roles of peptides in immunity.
}

It has been known for over 30 years that peptides are critical factors in mobilizing the immune system against foreign invaders. Since then, new knowledge about the generation and function of peptides in immunology has supported efforts to harness the immune system to treat disease. Yet, most of the highly successful treatments, including vaccines, have been made empirically, with little immunological insight. However, increased knowledge about the chemistry and pharmacological properties of antigenic and antimicrobial peptides as well as of the biology of antigen processing, presentation, and recognition by immune cells, has now enabled a more rational approach to vaccine and drug design. The Review, Perspective and Commentary articles in this issue highlight the role of peptides in both the innate and adaptive immune systems and outline how these key players can be used for therapy against infectious disease, cancer and autoimmune conditions.

Much progress has been made in defining the physiological roles of adaptive and innate immunity in meeting the challenges brought on by our environments. Adaptive immunity uses memory and specificity as a defense mechanism. Conversely, innate immunity is the first line shotgun approach of defense against infections and is exemplified by antimicrobial peptides, also known as host defense peptides (HDPs). HDPs are traditionally thought to be broad-spectrum antibiotic peptides that do not confer longlasting or protective immunity to the host. However, in a Perspective article (p. 776), Hilchie, Wuerth and Hancock review data that challenge the view that HDPs act primarily through cytotoxic mechanisms. For example, these peptides also have immunomodulatory activities, for instance in suppressing proinflammatory cytokines, which can more readily explain their ability to treat microbial infections. In addition, HDPs can affect the adaptive immune system, for instance in stimulating B- and T-cell responses. The study of HDPs and synthetic HDPs, termed innate defense regulator peptides, has led to the use of these peptides as vaccine adjuvants and in managing microbial burden during wound repair. These and other HDP therapies have motivated the development of new approaches for their sustained delivery as well as new economical approaches for achieving increased affinity and stability and also more efficient syntheses.

In adaptive immunity, peptides do not work alone. The Review article by Neefjes and Ovaa (p. 769) defines some key aspects of adaptive immunity and describes the path of a peptide from its initial generation by proteolytic processing to its presentation to immune cells by major histocompatibility complex (MHC) molecules. Adaptive immunity relies on the capacity of immune cells to distinguish between the body's own (self) antigens and unwanted invaders (nonself) and tumor cells. Key players are MHC-I and MHC-II that form a noncovalent complex with antigenic peptides and 'present' these peptides in the context of antigenpresenting cells to T cells of the immune system. MHC-I and MHC-II differ in structure and function as well in the length of the bound peptides.

The intricacies of peptide processing by the proteasome (for MHC-I peptides) and the endolysosome system (for MHC-II peptides) are poorly understood. For instance, the current paradigm for the genesis of MHC-I peptides suggests that viral, bacterial or native transformed proteins are cleaved by the proteasome before transport to the surface of antigen-presenting cells, where the complex can stimulate $\mathrm{CD} 8^{+} \mathrm{T}$ cells. However, a News $\&$ Views piece (p. 759) by Van Damme and Menschaert discusses recent evidence that short host-derived peptides can be trafficked to the MHC-I pathway. Certainly, gaining new insights into the mechanisms of the immune system will instruct us in new ways to exploit immune pathways to better treat disease.

The fact that there is a length difference in $\mathrm{MHC}-\mathrm{I}$ versus $\mathrm{MHC}-\mathrm{II}$ bound peptides is intriguing in light of a recent study showing that long peptides that represent an underprocessed protein antigen (that must be processed in the host) perform better than short MHC-compatible peptides that are delivered in human peptide vaccination strategies-specifically, in a widely watched trial aimed at preventing human papillomavirus-induced malignancies (http://www.nejm.org/doi/full/10.1056/ NEJMoa0810097). Apparently, we are unable to predict the appropriate peptides suitable for use in peptide vaccinationalthough we can recognize that defining the correct peptide fragment, with exact post-translational modifications (PTMs), is critical.

In addition to proteasomal processing, immune peptides undergo PTM such as glycosylation and citrullination that can modify their recognition by the immune system. A Review article by Boons (p. 761) focuses on the myriad roles of glycosylation in the immune system. Glycosylation has been implicated in nearly every aspect of adaptive immune activation. For instance, besides peptides themselves, many other immune components, including MHC proteins, and T-cell receptors, are glycosylated. Modification by glycosylation can render a 'self' peptide susceptible to being recognized as nonself and lead to autoimmune conditions such as type 1 diabetes and rheumatoid arthritis. Conversely, glycosylation has been shown in some cases to lead to immune escape (mutation that renders the peptide invisible to the immune system).

In a Commentary (p. 749), Flower also raises the issue of unpredictability of epitopes as a key issue in being able to engineer and manipulate the immune system. Defining useful B- and T-cell epitopes (that are recognized by $\mathrm{B}$ cells and $\mathrm{T}$ cells) has not been straightforward, and Flower argues that the obstacle is not in the lack of tools available, instead the real deficiency in prediction seems to lie in the complexity of the system. The Commentary further argues that we need a quantitative and predictive systems biology-style model of antigen presentation to incorporate such considerations as immunodominance (knowing which few of among the many possible antigenic peptides encoded by a pathogen is capable of eliciting a T-cell response) and immune escape. Certainly, gaining new molecularlevel insights into immune function at all stages of peptide processing, presentation and recognition are critically important as well.

Being able to gain new mechanistic insights into immune processes and to define and generate appropriate peptide antigens, in combination with medicinal chemistry approaches to improve peptide affinity, stability and delivery, are all areas where chemical biologists are poised to contribute. These necessary advances offer the promise of providing new vaccine strategies for infections and tumor immunology as well as antagonistic peptides for quenching autoimmune responses. 